Z. Klin. Chem. Klin. Biochem.

12. Jg. 1974 , S. $303-308$

\title{
Untersuchungen über die Abhängigkeit des Gesamtserumcholesterinspiegels der Ratte von Alter, Geschlecht und von der Jahreszeit
}

\author{
Von $A$. Metz, P. Donatsch und M. Madörin \\ Medizinisch-Biologische Forschung (Leitender Direktor: Dr. K. Saameli) SANDOZ AG, Basel (Schweiz)
}

(Eingegangen am 19. November 1973/24. Februar 1974)

\begin{abstract}
Es wird eine Modifikation der Technicon-Autoanalyzer-Methode N-37a zur Routinebestimmung von Gesamtcholesterin im Serum von Kleintieren beschrieben. Mit Hilfe dieses Verfahrens wurden über 3 Jahre Bestimmungen an Ratten verschiedenen Alters durchgeführt und die Abhängigkeit des Gesamtserumcholesterins von Alter, Geschlecht und von der Jahreszeit retrospektiv beurteilt. Dabei zeigt die Ratte deutlich geschlechtsspezifische Unterschiede im bekannten, altersbedingten Ansticg von Serumcholesterin. Eine saisonale Beeinflussung kann sicher an 2 Altersgruppen (14 und 21 Wochen) nachgewiesen werden. Bei einem älteren Kollektiv ( 34 Wochen) scheint sich diese Abhängigkeit zu vermindern.
\end{abstract}

\section{Studies on the total serum cholesterol concentration of the rat, and its dependence on age, sex and season}

A modification of the Technicon Autoanalyzer method N-37 a for the routine determination of total serum cholesterol in rodents is described. For a period of over three years this method was used for determining total serum cholesterol in rats. Results were subsequently analysed with respect to age and sex, as well as seasonal influences. The serum cholesterol was found to increase with age, and the sexes showed distinct differences in the rate of this increase. Obvious seasonal differences were found in 14 and 21-week old rats. In older populations ( 34 weeks) this seasonal ability for adaptation diminishes.

Das Verhalten des Serumcholesterinspiegels bei Mensch und Tier ist im Zusammenhang mit verschiedenen pathologischen Zuständen, wie z. B. Myokardschäden (1-3), Arteriosklerose (4-6) und Gallengangsaffektionen $(7,8)$, beschrieben worden. Die Suche nach Korrelationen hat wiederholt dazu geführt, daß man am gesunden Individuum die Abhängigkeit des Serumcholesterins von Alter und Geschlecht (7-11), von der Diät (12-14) und von der Jahreszeit (15-20) untersuchte.

In der vorliegenden Studie soll an einem größeren Rattenkollektiv das Verhalten des Serumcholesterinspiegels in Abhängigkeit von Alter, Geschlecht und von der Jahreszeit retrospektiv betrachtet und statistisch ausgewertet werden (t-Test).

\section{Material und Methode}

Das nachstehend beschriebene Autoanalyzersystem beruht im Prinzip auf der Methode von Levin und Zäk $(20,21)$. Damit diese Methode auch im Kleintierversuch angewendet werden konnte, mußten einige Änderungen durchgeführt werden, so daß vom technischen Standpunkt aus eine Methodenbeschreibung angebracht ist.

\section{Geräte}

Probennehmer, Pumpe, Heizbad (Technicon), Photometer (Eppendorf), Schreiber (W+W-1100, Kontron Zürich)

\section{Reagenzien}

Isopropanol (Merck) p. a. (Nr. 9634)

konz. Schwefelsäure $(D=1,84)$ p. a. (Merck) (Nr. 731)
Eisessig (Merck) p. a. (Nr. 90063)

Essigsäureanhydrid (Merck) p. a. (Nr. 42)

$\mathrm{FeCl}_{3}$, wasserfrei (Merck) p. a. (Nr. 3945)

Cholesterin (Merck) p. a. (Nr. 3670)

Herstellung der Lösungen

a) Eisen[III]-chloridlösung

$1 \mathrm{~g} \mathrm{FeCl}_{3}$ (wasserfrei) wird in 2,5 1 Eisessig aufgelöst und über Glaswolle filtriert, um etwaige unlösliche Rückstände $\mathrm{zu}$ entfernen.

b) Cholesterinreagenz

Gleiche Volumenteile Eisenchloridlösung und konzentrierte Schwefelsäure werden in der Kälte unter Rühren vermischt. In die kalte Reagenzlösung gibt man langsam 10\% des Volumens Essigsäureanhydrid unter Kühlung hinzu. Anmerkung: Die Lösung muß vor Gebrauch frisch hergestellt werden.

Standard-bzw. Testserum

Als Standard und gleichzeitig als Kontrolle der tierexperimentellen Analy senresultate wurden in den angegebenen Zeiten am Anfang bzw. am Ende jeder serienmäßigen Cholesterinbestimmung Versatol (Cosmopharm, Zürich) oder Monitrol (Merz u. Dade, Bern) verwendet $(25,50,75$ u. $100 \mu$ l Testserum/ml Isopropanol.

\section{Flußdiagram m}

für die Bestimmung von Gesamtcholesterin im Serum siehe Abbildung 1.

Ausführung der Bestimmung des Gesam tcholesterins Für die Cholesterinbestimmung werden $50 \mu 1$ Serum in $1 \mathrm{ml}$ Isopropanol 30 Minuten mechanisch extrahiert und der Niederschlag abzentrifugiert. Das Gesamtcholesterin bestimmt 


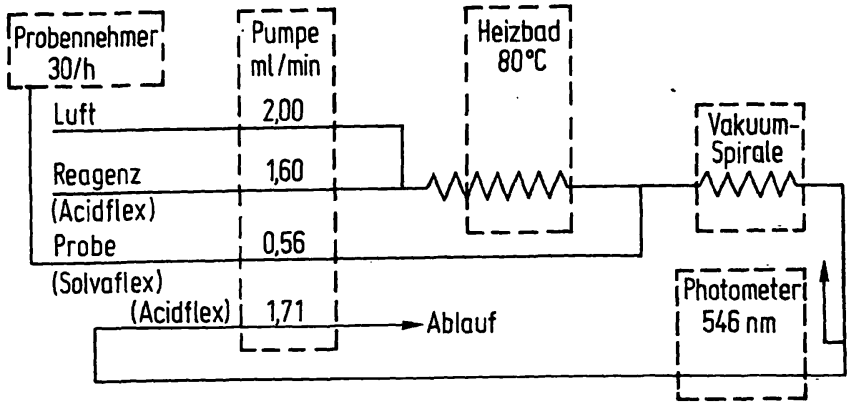

Abb. 1. Flußdiagramm Gesamtcholesterin

Anmerkung: Probeentnahmezeit 35 s, Schichtdicke der Küvette $1 \mathrm{~cm}$, Waschflüssigkeit: Isopropanol

man darauf im Isopropanol-Extrakt nach der eben beschriebenen, modifizierten Autoanalyzermethode. Der apparative Fehler des Systems liegt bei 0,54\%. Unter Einbeziehung des Extraktionsschrittes erhöht sich der mittlere Bestimmungsfehler auf $1,2 \%$.

\section{Tierexperimentelles Verfahren}

Alle Untersuchungen wurden an Wistar Ratten [OFA, aus IFFA-Credo oder Eigenzucht Sandoz, SPF-Tiere (spezifiziert pathogenfreie Tiere)] durchgeführt. Die Tiere waren in vollklimatisierten Räumen untergebracht (Raumtemperatur $24 \pm 1{ }^{\circ} \mathrm{C}$, Luftfeuchtigkeit etwa $50-60 \%$, konstanter TagNacht-Rhythmus durch künstliches Licht, 15 fache Luftumwälzung pro Stunde). Für die Untersuchung wurden Kontrolltiere aus 76 verschiedenen Versuchen der letzten drei Jahre herangezogen. Während der Versuchsdauer (8-100 Wochen) standen die Tiere nicht unter SPF-Bedingungen. Sie lebten einzeln in Makrolon-Wannen (Typ II bzw. Typ III). Die Ratten hatten freien Zugang zu Wasser und Nafag-Futter $-194 . *$ )

*) Angaben über die Zusammensetzung des Futters können auf Wunsch bei den Autoren eingeholt werden. (Hersteller: Fa. Nafag, Gossau, Schweiz).
Je nach der toxikologischen Versuchsdauer wurde den aus verschiedenen Würfen stammenden Kontrolltieren nach 8, 14, $21,34,60$ und 100 Wochen je einmal Blut entnommen. Die Blutentnahme erfolgte retroorbital unter leichter Äthernarkose jeweils vormittags zwischen 8 und $10 \mathrm{Uhr}$. Das Blut wurde in verschließbaren Plastikröhrchen (Eppendorf-Röhrchen) eine Stunde bei Raumtemperatur belassen. Dann wurde 5 Minuten bei $4000 \mathrm{~g}$ zentrifugiert (Blutzentrifuge Ecco Quick) und das Serum gewonnen. . '

\section{Resultate}

Im Rahmen toxikologischer Studien sammeln wir $\mathrm{zu}$ statistischen Zwecken seit Jahren Gesamtcholesterinwerte bei Kontroll-Ratten $(q+\delta)$ verschiedenen Alters. Um Jahresdurchschnittswerte berechnen zu können, haben wir das vorliegende Tiermaterial nach Untersuchungsjahren aufgeschlüsselt. In Tabelle 1 sind die Jahresmittel und die prozentuale Zusammensetzung der einzelnen Altersgruppen des Jahreskollektives dargestellt.

Diese Resultate (Tab. 1) zeigen, daß der Mittelwert selbst ziemlich konstant bleibt, die Standardabweichung jedoch mit den Jahren deutlich größer wird. Dies hat uns bewogen, sämtliche Cholesterinwerte der dreijährigen Sammelperiode nach Alter und Geschlecht zu gliedern, die Werte auf ihre Normalverteilung żu untersuchen und etwaige auftretende Differenzen statistisch mit Hilfe des t-Testes (nach Student) zu prüfen.

Wie die Verteilungsstudien ( $\chi^{2}$-Test) in Tabelle 2 zeigen, ist nach Auftrennung des Rattenkollektives nach Alter und Geschlecht eine Normalverteilung gegeben. Die Aufteilung in mehrere Altersbereiche ergibt nur in den angegebenen Kombinationen eine Normalverteilung; alle übri-

Tab. 1. Jahresmittelwerte von Gesamtcholesterin bei Ratten verschiedener Altersstufen und prozentualer Anteil derselben am Jahreskollektiv (Geschlechtsverhältnis $1 \mathrm{zu} 1$ )

\begin{tabular}{|c|c|c|c|c|c|c|c|c|}
\hline \multirow[t]{2}{*}{ Jahr } & \multirow[t]{2}{*}{$\mathbf{n}$} & \multirow[t]{2}{*}{$\overline{\mathbf{x}}( \pm \mathrm{s})[\mathrm{mmol} / \mathrm{l}]$} & \multicolumn{6}{|c|}{ Alterszusammensetzung der Tiere in \% } \\
\hline & & & 8 & 14 & 21 & 34 & 60 & 100 Wochen \\
\hline $\begin{array}{l}1970 \\
1971 \\
1972\end{array}$ & $\begin{array}{l}340 \\
158 \\
362\end{array}$ & $\begin{array}{l}2,35( \pm 0,46) \\
2,48( \pm 0,80) \\
2,53( \pm 1,03)\end{array}$ & $\begin{array}{r}0 \\
9 \\
16\end{array}$ & $\begin{array}{l}48 \\
41 \\
37\end{array}$ & $\begin{array}{l}33 \\
36 \\
33\end{array}$ & $\begin{array}{r}19 \\
5 \\
8\end{array}$ & $\begin{array}{l}0 \\
9 \\
0\end{array}$ & $\begin{array}{l}0 \\
0 \\
6\end{array}$ \\
\hline
\end{tabular}

Tab. 2. Verteilungsstudien bei Ratten verschiedenen Alters und Geschlechts ( $x^{2}$-Test)

\begin{tabular}{|c|c|c|c|c|c|c|c|}
\hline Alter & & Verteilung & & Verteilung & & Veṛteilung & \\
\hline 8 & $\delta$ & normal & q & normal & $6+9$ & nicht normal ) & \\
\hline 14 & o & normal & क & normal & $8+q$ & nicht normal & Exżess \\
\hline 21 & $\delta$ & normal & 9 & normal & $0+q$ & nicht normal & \\
\hline 34 & $\delta$ & normal & q & normal & $0+\infty$ & normal & \\
\hline 60 & 0 & - & \% & - & $0+q$ & - & \\
\hline 100 & $\delta$ & - & $\%$ & - & $\delta+8$ & - & \\
\hline $8-100$ & o & nicht normal & $\%$ & nicht normal & $0+q$ & nicht normal & \\
\hline $8+14$ & o & normal & \% & normal & $0+8$ & nicht normal & \\
\hline $14+21$ & o & normal & & leichte Abweich. (Exzess) & $0+8$ & nicht normal & \\
\hline $21+34$ & đ & normal & 8 & normal & $8+q$ & nicht normal & \\
\hline $34+60$ & ठ & normal & .8 & normal & $0+8$ & nicht normal & \\
\hline
\end{tabular}

Bemerkung: Die Berechnungen basieren auf den Tierzahlen, die in Tabelle 3 angegeben sind.

- $=\mathrm{zu}$ wenig Werte 
Tab. 3. Abhängigkeit des Gesamtserumcholesterins von Alter und Geschlecht bei Ratten.

\begin{tabular}{|c|c|c|c|c|c|c|c|c|c|c|}
\hline \multirow{2}{*}{$\begin{array}{l}\text { Alter } \\
\text { in } \\
\text { Wochen }\end{array}$} & \multicolumn{3}{|c|}{ Männliche Tiere } & \multicolumn{4}{|c|}{ Weibliche Tiere } & \multicolumn{3}{|c|}{ Männliche + Weibliche Tiere } \\
\hline & $\begin{array}{l}\text { Tierzahl } \\
\text { n }\end{array}$ & $\begin{array}{l}\text { Cholesterin } \\
{[\mathrm{mmol} / \mathrm{l}]} \\
(\overline{\mathbf{x}} \pm \mathrm{s})\end{array}$ & $\begin{array}{l}\text { Signifikanz } \\
\text { p }\end{array}$ & $\begin{array}{l}\text { Tierzahl } \\
\text { n }\end{array}$ & $\begin{array}{l}\text { Cholesterin } \\
{[\mathrm{mmol} / \mathrm{l}]} \\
(\overline{\mathbf{x}} \pm \mathrm{s})\end{array}$ & $\begin{array}{l}\text { Signifikanz } \\
\text { p }\end{array}$ & $\begin{array}{l}\text { Signifikanz } \\
\text { Po/Q. }\end{array}$ & $\begin{array}{l}\text { Tierzahl } \\
\mathrm{n}\end{array}$ & $\begin{array}{l}\text { Cholesterin } \\
{[\mathrm{mmol} / 1]} \\
(\overline{\mathrm{x}} \pm \mathrm{s})\end{array}$ & $\begin{array}{l}\text { Signifikanz } \\
\text { p }\end{array}$ \\
\hline 8 & 33 & $1,91 \pm 0,44$ & & 34 & $2,35 \pm 1,01$ & & $<0,023$ & 67 & $2,14( \pm 0,78)$ & - \\
\hline 14 & 145 & $2,25 \pm 0,44$ & $\begin{array}{l}<0,0005 \\
<0,751\end{array}$ & 152 & $2,48 \pm 0,49$ & $\begin{array}{l}<0,329 \\
<0,698\end{array}$ & $<0,0005$ & 297 & $2,38( \pm 0,47)$ & $\begin{array}{l}<0,005^{*} \\
<0,663\end{array}$ \\
\hline 21 & 163 & $2,27 \pm 0,59$ & $<0,001$ & 163 & $2,51 \pm 0,70$ & $<0,435$ & $<0,003$ & 326 & $2,38( \pm 0,65)$ & $<0,003$ \\
\hline 34 & 50 & $2,64 \pm 0,65$ & $<0,0005$ & 53 & $2,58 \pm 0,52$ & $<0,0003$ & $<0,609$ & 103 & $2,61 \pm 0,59$ & $<0,0005$ \\
\hline 60 & 10 & $5,17 \pm 1,50$ & $<0,091$ & 9 & $3,54 \pm 0,62$ & $<0,044$ & $<0,008$ & 19 & $4,39 \pm 1,42$ & $<0,025$ \\
\hline 100 & 10 & $6,30 \pm 1,29$ & & 10 & $4,78 \pm 1,60$ & & $<0,032$ & 20 & $5,53 \pm 1,60$ & \\
\hline
\end{tabular}

$\overline{\mathrm{x}} \pm \mathrm{s} \quad$ : Mittelwert \pm Standardabweichung

p : Signifikanz des Unterschiedes zur jeweiligen nächst niedrigeren Altersgruppe.

$\mathrm{P}_{\delta} / \mathrm{Q}$ : Signifikanz des Unter schiedes zwischen Männchen und Weibchen der gleichen Altersstufe.

*) : Obwohl die erforderlichen Voraussetzungen (Normalverteilung) für den t-Test nicht in jedem Falle gegeben sind, erhält man bei den gepoolten Werten ähnliche Signifikanzen (s. Tab. 2).

gen denkbar möglichen Kombinationen, vor allem Kombinationen mit mehreren Altersstufen, zeigten keine Normalverteilung (eine zusätzliche, ausführlichere Studie hierüber, die diese Routinebefunde bestätigt, ist in Bearbeitung).

Die Resultate in Tabelle 3 zeigen bei den männlichen Tieren bereits ab der 21 . Lebenswoche, bei den weiblichen erst ab der 34 . Woche einen deutlichen und statistisch signifikanten Anstieg des Cholesterins. Dabei ist bemerkenswert, daß bis zum Alter von etwa 30 Wochen das weibliche gegenüber dem männlichen Tier einen signifikant höheren Cholesterinspiegel aufweist.

Infolge steileren Anstieges des Serumcholesterinspiegels bei der männlichen Ratte zwischen 30 und 60 Wochen überschneiden sich ab der 34. Woche die Cholesterinwerte beider Geschlechter. Von diesem Zeitpunkt an sind die Mittelwerte bei den männlichen Tieren signifikant höher als die der weiblichen.

Außerdem wird aus den Ergebnissen auch ersichtlich, daß die Zunahme der Standardabweichung mit dem altersbedingten Cholesterinanstieg korreliert.

Auf Grund der eingangs zitierten Arbeiten über jahreszeitliche Schwankungen des Serumcholesterins bei Mensch und Tier, haben wir unsere Daten auf monatliche Schwankungen in Abhängigkeit zum Alter untersucht. Wie aus Tabelle 4 und Abbildung 2 hervorgeht, zeigen sich während der Frühjahrs- und Spätsommermonate bei der Ratte deutliche signifikante Veränderungen der Serumcholesterinwerte. Global beurteilt treten in der Frühjahrszeit durchweg die niedrigsten Werte auf. Bei den jüngeren Altersstufen (14 und 21 Wochen) steigen die Werte während der Sommermonate etwas an und erreichen ein Maximum im Monat September. Es folgt ein Minimum, dann steigen die Werte mit beginnender kälterer Jahreszeit weiter an. Bei den 34wöchigen Ratten sind diese saisonalen Veränderungen nicht mehr so deutlich.

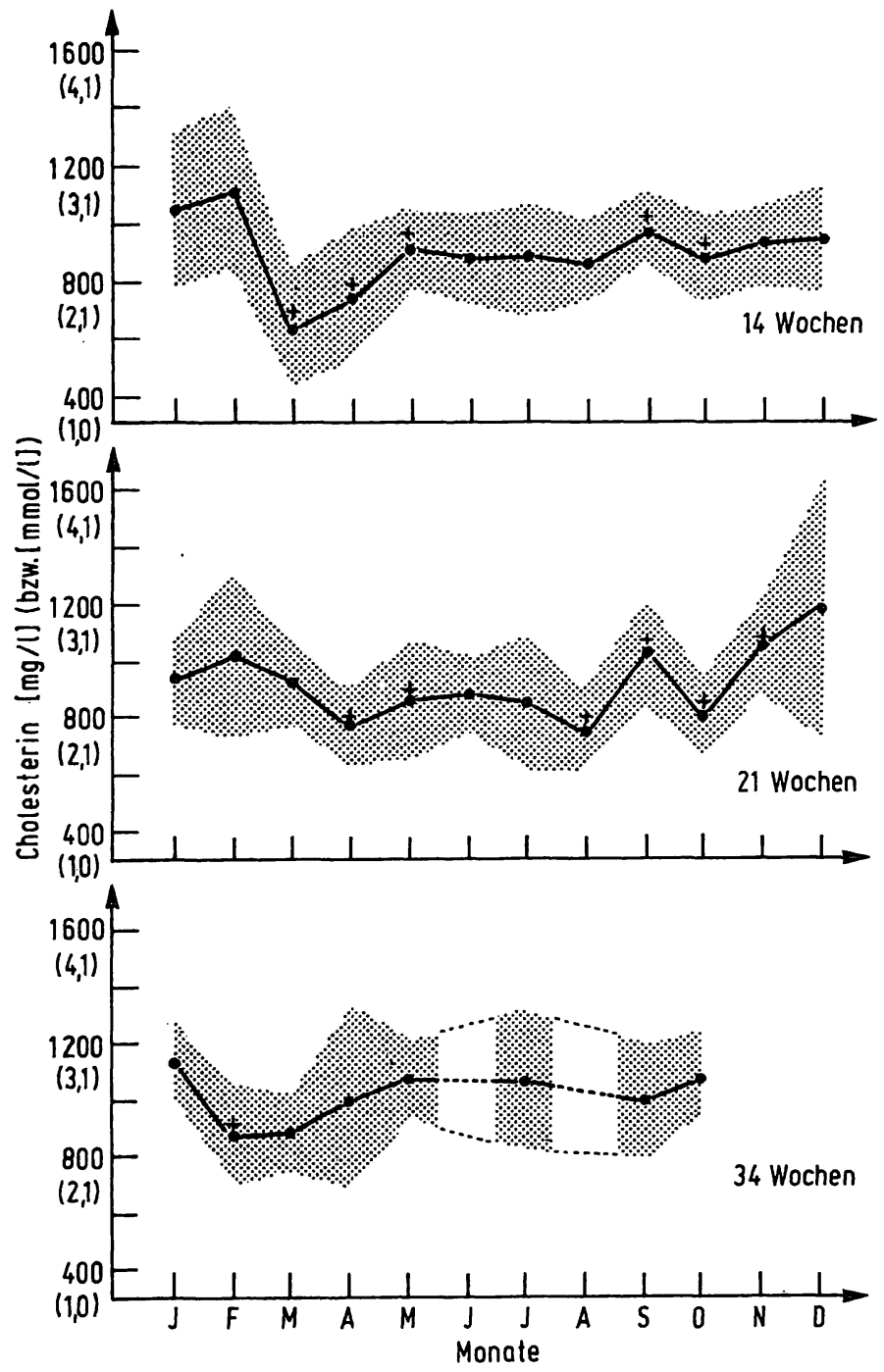

Abb. 2. Saisonale Abhängigkeit des Gesamtserumcholesterins bei Ratten $(\delta+\%)$ verschiedener Altersstufen

+ Signifikanz des Unterschiedes zum jeweiligen Vormonat $(p<0,05)$

Streubereich $( \pm$ s) gerastert

Abszisse: Kalendermonate, Ordinate: Cholesterinkonzentration, $\mathrm{mg} / \mathrm{l}$; Angaben in Klammern $=\mathrm{mmol} / \mathrm{l}$ 
Tab. 4. Monatliche Schwankungen des Gesamtserumcholesterins (mmol/1) bei Ratten $(\varnothing+\varnothing)$ verschiedener Altersstufen

\begin{tabular}{|c|c|c|c|c|c|c|c|c|c|}
\hline \multirow{2}{*}{$\begin{array}{l}\text { Alter } \\
\text { Monat }\end{array}$} & \multicolumn{2}{|c|}{14 Wochen } & \multirow{2}{*}{$\begin{array}{l}\text { Signifikanz } \\
\text { p }\end{array}$} & \multicolumn{2}{|c|}{21 Wochen } & \multirow{2}{*}{$\begin{array}{l}\text { Signifikanz } \\
\text { p }\end{array}$} & \multicolumn{2}{|c|}{34 Wochen } & \multirow{2}{*}{$\begin{array}{l}\text { Signifikanz } \\
\text { p }\end{array}$} \\
\hline & $\mathrm{n}$ & $\bar{x} \pm s$ & & $\mathrm{n}$ & $\bar{x} \pm s$ & & $\mathbf{n}$ & $\bar{x} \pm s$ & \\
\hline Januar & 29 & $2,66 \pm 0,68$ & $<0,24$ & 30 & $2,38 \pm 0,40$ & $<0,09$ & .9 & $2,92 \pm 0,37$ & $<0,001^{*}$ \\
\hline Februar & 61 & $2,84 \pm 0,73$ & $<0,001^{*}$ & 40 & $2,61 \pm 0,69$ & $<0,15$ & 10 & $2,25 \pm, 0,45$ & $<0,95$ \\
\hline März & 40 & $1,60 \pm 0,51$ & $<0,02 *$ & 20 & $2,38 \pm 0,40$ & $<0,001^{*}$ & 8 & $2,25 \pm 0,34$ & $<0,29$ \\
\hline April & 59 & $1,89 \pm 0,59$ & $<0,001 *$ & 29 & $1,96 \pm 0,36$ & $<0,04 *$ & 20 & $2,58 \pm 0,85$ & $<0,56$ \\
\hline Mai & 19 & $2,35 \pm 0,37$ & $<0,35$ & 28 & $2,20 \pm 0,50$ & $<0,71$ & 8 & $2,76 \pm 0,36$ & $<0,90$ \\
\hline Juni & 40 & $2,25 \pm 0,38$ & $<0,74$ & 9 & $2,27 \pm 0,34$ & $<0,67$ & & - & \\
\hline Juli & 40 & $2.27 \pm 0,56$ & $<0,67$ & 40 & $2,20 \pm 0,60$ & $<0,06$ & 30 & $2,74 \pm 0,63$ & \\
\hline August & 10 & $2,20 \pm 0,37$ & $<0,01^{*}$ & 20 & $1,89 \pm 0,39$ & $<0,001 *$ & & - & $<0,43$ \\
\hline September & 46 & $2,51 \pm 0,34$ & $<0,01 *$ & 30 & $2,64 \pm 0,47$ & $<0,001 *$ & 9 & $2,56 \pm 0,52$ & $<0,32$ \\
\hline Oktober & 20 & $2,25 \pm 0,42$ & $<0,31$ & 20 & $2,04 \pm 0,40$ & $<0,001 *$ & 10 & $2,76 \pm 0,38$ & \\
\hline November & 30 & $2,38 \pm 0,39$ & $<0,63$ & 30 & $2,69 \pm 0,48$ & $<0,16$ & & - & \\
\hline Dezember & 40 & $2,43 \pm 0,46$ & & 29 & $3,02 \pm 1,20$ & & & - & \\
\hline
\end{tabular}

$\mathrm{n} \quad=$ Anzahl der Tiere (Zur Ergänzung wurden Tiere aus Versuchen von 1973 noch einbezogen)

$\overline{\mathrm{X}} \pm s=$ Mittelwerte und Standardabweichung (in mmol/l)

* $\quad$ = Signifikanz des Unterschiedes zum jeweiligen Vormonat $(p<0,05)$

- $\quad$ Keine Werte vorhanden

Tab. 5. Statistische Prüfung auf saisonale Unterschiede bei Ratten verschiedener Altersstufen (T-Verteilung und P-Integral).

\begin{tabular}{|c|c|c|c|c|c|c|c|c|}
\hline \multirow[t]{2}{*}{ Alter } & \multirow[t]{2}{*}{ Geschlecht } & \multicolumn{3}{|c|}{$\begin{array}{l}\text { Periode mit niederen Cholesterinspiegeln } \\
\text { (in mmol/l) }\end{array}$} & \multicolumn{3}{|c|}{$\begin{array}{l}\text { Periode mit höheren Cholesterinspiegeln } \\
\text { (in mmol/l) }\end{array}$} & \multirow{2}{*}{$\begin{array}{l}\text { Signifikanz des } \\
\text { Unterschiedes } \\
\text { zwischen bei- } \\
\text { den Perioden }\end{array}$} \\
\hline & & Saison & $\mathrm{n}$ & $\bar{x} \pm s$ & Saison & $\mathbf{n}$ & $\bar{x} \pm s$ & \\
\hline 14 & 0 & März - Juli & 99 & $2,09 \pm 0,44$ & August - Februar & 118 & $2,38 \pm 0,44$ & $p=0,0001$ \\
\hline 21 & o & März - Juli & 63 & $2,07 \pm 0,52$ & August - Februar & 100 & $2,40 \pm 0,65$ & $\mathrm{p}=0,001$ \\
\hline 34 & 0 & März - Juli & 32 & $2,69 \pm 0,72$ & August - Februar & 18 & $2,56 \pm 0,57$ & $p=0,547$ \\
\hline 34 & o & Februar - März & 8 & $2,02 \pm 0,34$ & April - Januar & 42 & $2,76 \pm 0,65$ & $p=0,003$ \\
\hline 14 & \$ & März - Juli & 99 & $2,38 \pm 0,44$ & August - Februar & 118 & $2,58 \pm 0,52$ & $p=0,01$ \\
\hline 21 & $\varphi$ & März - Juli & 63 & $2,27 \pm 0,47$ & August - Februar & 99 & $2,64 \pm 0,78$ & $p=0,002$ \\
\hline 34 & \% & März - Juli & 34 & $2,58 \pm 0,62$ & August - Februar & 20 & $2,66 \pm 0,41$ & $p=0,640$ \\
\hline 34 & ९ & Februar - März & 10 & $2,43 \pm 0,36$ & April - Januar & 44 & $2,64 \pm 0,57$ & $\mathrm{p}=0,269$ \\
\hline 14 & $o+q$ & März - Juli & 198 & $2,03 \pm 0,58$ & August - Februar & 236 & $2,55 \pm 0,57$ & $p=0,0001 *$ \\
\hline 21 & $\delta+9$ & März - Juli & 126 & $2,18 \pm 0,50$ & August - Februar & 199 & $2,52 \pm 0,73$ & $\mathrm{p}=0,0001^{*}$ \\
\hline 34 & $0+9$ & März - Juli & 66 & $2,64 \pm 0,66$ & August - Februar & 38 & $2,61 \pm 0,49$ & $p=0,853$ \\
\hline 34 & $0+9$ & Februar - März & 18 & $2,25 \pm 0,39$ & April - Januar & 86 & $2,71 \pm 0,61$ & $p=0,004$ \\
\hline
\end{tabular}

* Keine Normalverteilung (Excess, siehe Tab. 2)

Um eindeutig zu belegen, daß es sich hierbei um sogenannte saisonale Veränderungen handelt, haben wir die zusammenhängenden Frühjahrs- und Sommerperioden mit niederen Cholesterinspiegeln nach Geschlecht getrennt zusammengefaßt und die Werte mit den übrigen kälteren Jahreszeiten verglichen. Die Befunde zeigten eine statistisch gesicherte, saisonale Veränderung des Serumcholesterinspiegels.

\section{Diskussion}

Die Bestimmung des Gesamtcholesterins im Serum nach Levin und Zak darf heute als eine der zuverlässigsten und einfachsten Routinemethoden angesehen werden $(3,23)$. Bei der Modifikation der Technicon-Autoanalyzer-Methode N-37 a zur Anwendung am Kleintier stellte sich heraus, daß durch Zusatz von etwas Essigsäurean- 
hydrid zum Reagenz und durch Vorwärmen desselben auf $80^{\circ}$ die Betriebssicherheit verbessert und die Empfindlichkeit der Methode noch um etwa $100 \%$ gesteigert werden kann. Nach unseren Erfahrungen liegen bei der automatischen Bestimmung, gleichbleibende Reagenzienqualität vorausgesetzt, die Hauptstörfaktoren im Feuchtigkeitsgehalt des Reaktionsgemisches und in Temperaturschwankungen.

Die vorliegenden Ergebnisse, d. h. Zunahme der Standardabweichung und die geschlechtsspezifische Erhöhung des Gesamtcholesterins mit zunehmendem Alter, machen es unseres Erachtens notwendig, bei der konventionell gehaltenen Ratte einen nach Lebensphasen und Geschlecht gestaffelten Normalbereich zu schaffen. (siehe Tab. 2).

Ein nach Altersstufen gestaffelter Normbereich hättè den Vorteil, daß man durch Vergleich mit der jüngeren, der gleichen und der nächst höheren Altersstufe Veränderungen frühzeitig erkennen und altersbedingte und pathologische Abweichungen besser differenzieren könnte. Daß auch beim Menschen eine ähnliche Problematik besteht, geht aus Arbeiten hervor $(1,8,11$, $15)$, in denen gezeigt wird, daß der Cholesterinspiegel ähnlich wie bei der Ratte geschlechtsspezifisch ansteigt (steiler Anstieg beim Mann zwischen 30. und 50. Lebensjahr, dann Abflachung des Anstieges; bei der Frau kontinuierliche Cholesterinerhöhung über beinahe das ganze Leben). Dieses Entwicklungsbild des Menschen ist mit dem der Ratten altersmäßig grob vergleichbar, wenn man den allgemein üblichen Altersvergleichsfaktor Ratte-Mensch von 30-35 anwendet. Die gefundenen saisonalen Schwankungen des Cholesterinspiegels bei Ratten der Altersgruppen 14 und 21 Wochen finden ihre Parallele neben anderen in den Untersuchungen von Pincherle (15), Thomas et al (16), Doyle et al (17), Carlson und Lindstedt (18) am Menschen und von Kritschewsky und seiner Gruppe (19) am Affen, wo ebenfalls während der
Frühjahrs- bzw. Sommermonate niedrigere Cholesterinwerte gefunden wurden.

Bei der Ratte werden ähnliche saisonale Abweichungen nach unserem Wissen nur von Thorp (20) beschrieben. Nach unseren Untersuchungen scheint jedoch bei der Ratte mit zunehmendem Alter die saisonale Adaption verloren zu gehen. Letz teres Ergebnis könnte unter Umständen einen Hinweis auf die Befunde der Arbeitsgruppen von Van Houte (1) und von Samuel (5) geben, welche an Patientenkollektiven den Nachweis saisonaler Schwankungen nicht erbringen konnten. Über die Ursache dieser Schwankungen kann nach unseren Untersuchungen keine Aussage gemacht werden. Wir können lediglich Umweltfaktoren wie jahreszeitliche Temperaturschwankungen, Änderungen im Tag- und Nachtrhy thmus, sowie Beeinflussung durch Diätänderung als Ursache ausschließen. Möglicherweise trifft auch bei der Ratte die von Doyle et al (17) für saisonale Schwankungen beim Menschen gegebene Erklärung zu, welche sie mit jahreszeitlichen Änderungen der Schilddrüsenfunktion in Zusammenhang bringen. Diese Annahme ist jedoch in unserem Falle nur dann berechtigt, wenn wir weitere, bisher nicht eliminierbare exogene und endogene Faktoren in Betracht ziehen (z. B. Änderung des generellen Hormongleichgewichtes, des Sexualverhaltens, oder sonstige biophysikalische Faktoren). Praktisch bedeutsam könnten diese Befunde bei der Beurteilung von cholesterinsenkenden Medikamenten sein. Die beschriebenen Spontanschwankungen sollten schon bei der Planung derartiger experimenteller Studien (bei Tier und Mensch) berücksichtigt werden.

\section{Danksagung}

Unseren technischen Mitarbeitern, den Herren R. Peter, $H$. Rothen und G. Lützelschwab möchten wir für Ihren Einsatz bei der Entwicklung der Methode und für die Ausführung der Bestimmungen danken.

\section{Literatur}

1. van Houte, O. \& Kesteloot, H. (1972), Acta Cardiol. (Bruxelles), 27, 527-564.

2. Dayton, S. (1971), Fed. Proc. Fed. Amer. Soc. Exp. Biol. 30, 849-856.

3. Boehm, Edith \& Reinhard, H. (1970), Zentralbl. f. Veterinärmed., 17, 536-549.

4. Kritschewsky, D. (1970), Amer. J. Clin. Nutr. 23, 1105-1110.

5. Samuel, P., Lieberman, S., Shmase, F. S., Meilman, E., \& Touflexis, G. (1970), Amer. J. Clin. Nutr. 23, 178-188.

6. Hill, E. G. (1972), Lancet I, 1286-1287.

7. Werner, M., Tolls, J., Hultin, J., \& Mellecker, J. (1970), diese Z., 8, 105-115.

8. Patterson, D. (1972), Lancet I, 393-399.
9. Hrouza, Z. \& Wachtlová, M. (1969), Exp. Geront, 4, 245-250.

10. Das, B. (1967), Gerontologia, 13, 227-245.

11. Hallberg, L., Högdahl, A., Svanborg, A. \& Vikrot, O. (1966), Acta Med. Scand., 180, 697-707.

12. McGandy, R., Hall, Barbara, Ford, Cynthia \& Stare, F. (1972), Amer. J. Clin. Nutr., 25, 61-66.

13. Greenberg, L. (1970), Amer. J. Clin. Nutr., 23, 1101-1104.

14. Cayen, M. (1969), Biochim. Biophys. Acta 187, 546554.

15. Pincherle, G. (1971), J. Cluron. Dis., 24, 289-297.

16. Thomas, C., Halljes, H. \& Eisenberg, F. (1969), Ann. Int. Med. 54, 413. 
11 Imigle. J., Kinch. Sandis a Hroun. D (1965). 1. Chron. Dis. 18.657 het

is Calunn. I I Inditrdt. S. (1968). Acta Mcd. Scand. Supul 193. 13: Talcl A77.

10. Kelluhiusky, D. Jallemsky, J., Shapiso, J., Kitt. ishers wh, I Nau. P. (1967), in the Haboon in Mrdical Rescasch (lid Vagtborg. II.). Vol. 2. p. 547 \$57. Austin I'neveruty of Teras Prose
20. Thorp. J. M. (1963). in The Control of Lipid Metabolium (Girant. J. K., cd.) p. 163-169, Academic Prese, New York. 21. Zlatkir, A., Zak, B. \& Boyle, A. (1953), J. Lab. Clin. Bied. 41.486.

22. Levin, J. Zak, B. (1964), Clin. Chim. Acta 10, 381 - 384.

23. Mueller, G. (1969), Das klin. Luboratorium 1944-1951.

Di. A. Metz

SANDOZ A. G. Pharm. Dept. CH 4002 Basel 